\title{
Criminologie
}

\section{La notion de dangerosité, maladie infantile de la criminologie}

\section{Christian Debuyst}

Volume 17, numéro 2, 1984

La dangerosité, un débat à poursuivre

URI : https://id.erudit.org/iderudit/017197ar

DOI : https://doi.org/10.7202/017197ar

Aller au sommaire du numéro

Éditeur(s)

Les Presses de l'Université de Montréal

ISSN

0316-0041 (imprimé)

1492-1367 (numérique)

Découvrir la revue

Citer cet article

Debuyst, C. (1984). La notion de dangerosité, maladie infantile de la criminologie. Criminologie, 17(2), 7-24. https://doi.org/10.7202/017197ar

\section{Résumé de l'article}

The accent is first of all on the fact (point 1) that the conception of dangerousness is a criminological construct based on the establishment of a causal model within the framework of an objective that is sought. It might be said that this objective is the control of criminal activities and behaviour of groups likely to threaten the social order.

It may be said that this point of view (point 2) makes any multidisciplinary approach relatively gratuitous because it does not allow the different disciplines to carry through their own logic.

The problem is (point 3) to know how to get rid of this notion without criminology losing its justification and the criminologist/practitioner his usefulness. 


\section{LA NOTION DE DANGEROSITÉ, MALADIE INFANTILE DE LA CRIMINOLOGIE Christian Debuyst*}

The accent is first of all on the fact (point 1) that the conception of dangerousness is a criminological construct based on the establishment of a causal model within the framework of an objective that is sought. It might be said that this objective is the control of criminal activities and behaviour of groups likely to threaten the social order.

It may be said that this point of view (point 2) makes any multidisciplinary approach relatively gratuitous because it does not allow the different disciplines to carry through their own logic.

The problem is (point 3) to know how to get rid of this notion without criminology losing its justification and the criminologist/practitioner his usefulness.

\section{INTRODUCTION}

Le problème de la dangerosité est sans doute l'une des questions les plus embarrassantes, parce qu'il est extrêmement difficile, à son propos, de ne pas tomber dans les redites et de ne pas reprendre un débat dans lequel les positions d'un chacun se trouvent réaffirmées sans que des éléments neufs ne viennent s'ajouter aux arguments et aux résultats de recherche qui déjà avaient été présentés".

Dans cet article, dès lors, nous pourrions difficilement éviter les redites. Nous voudrions néanmoins les rendre les plus cohérentes possibles par rapport à un certain nombre de problématiques que nous essayerons d'exprimer clairement. Nous devons en effet nous demander si le thème de la dangerosité, en criminologie clinique, est aussi central qu'on ne le dit. Le fait d'avoir déjà pris à son propos une distance ne résulte nullement d'une peur (peur de quoi? d'une sorte de «bon sens» criminologique?) comme semble le dire l'analyse critique faite dans cette revue des interventions présentées au cours du colloque qui s'est déroulé sur ce thème à Louvain-la-Neuve ${ }^{2}$. Y aurait-il peur de la dangerosité et de son utilisation, ou plutôt, la question n'est-elle pas de savoir si le fait d'avoir

* Professeur à l'École de criminologie de l'Université catholique de Louvain.

1. Voir les deux colloques organisés par le Centre international de criminologie clinique à Gênes (1981) et à Messine (1982) ainsi que le débat paru dans Déviance et société, 1981, vol. 4, n'4.

2. Christian Debuyst et al. (1981), Dangerosité et justice pénale, Ambiguité d'une pratique, Paris-Genève, Éditions Médecine et Hygiène, "Déviance et société". 
posé le problème de la délinquance à travers cette notion ne l'a pas enfermé dans une forme d'approche dont apparaissent maintenant les inconvénients et les limitations.

En introduisant ces quelques notes, nous ne nous perdrons pas dans la recherche d'une définition. Nous nous contenterons de dire, d'une manière commode, que la dangerosité est la probabilité que présente un individu de commettre une infraction, avec sans doute la limitation traditionnelle (en elle-même discutable) voulant que cette infraction soit une infraction contre les personnes et contre les biens ${ }^{3}$. Nous pourrions ajouter en disant que la dangerosité est la probabilité que présente une situation de donner lieu à des comportements de ce genre. Nous verrons plus loin pourquoi nous élargissons ainsi la notion, mais le problème ne nous paraît cependant pas se situer à ce niveau. Pour engager la discussion, nous préférons partir d'une question qui pourrait d'ailleurs constituer un test : la définition de la dangerosité établit-elle une équivalence entre les deux termes : dangerosité $=$ probabilité d'un comportement délinquant ou d'une récidive. Peut-on inverser les propositions et dire qu'une probabilité de récidive (ou qu'une probabilité de comportement délinquant) doit nécessairement se traduire en termes de dangerosité? Est-ce la seule manière d'aborder cette réalité?

Cette question est importante et n'est nullement une tautologie. Ce qui importe au criminologue, c'est effectivement d'aborder cette probabilité de récidive que quelqu'un présente dans un domaine déterminé, ou en d'autres termes, de s'interroger sur le maintien d'un comportement délinquant. Est-ce que le fait de transcrire cette préoccupation réelle en termes de dangerosité constitue la manière la plus adéquate de l'aborder correctement? Est-ce de cette manière que la criminologie est susceptible d'atteindre un statut scientifique, ou ne s'agit-il pas d'une manière de poser la question qui serait tributaire d'un moment du développement de la criminologie (sa maladie infantile) qui, à l'heure actuelle, suscite plus de difficultés qu'elle n'en résout dans ce qu'on pourrait appeler la recherche d'une certaine vérité et même d'une certaine efficacité?

C'est la question centrale que dans cet article, nous voudrions nous poser et à partir de laquelle nous chercherons à ordonner les différents points que nous aborderons. Très globalement, nous dirons que ces points seront de deux ordres : les premiers viseront à montrer que la notion de dangerosité, si nous l'utilisons comme outil scientifique, risque de nous engager dans de nombreux pièges. Nous avons affirmé dans le passé que

3. Voir la discussion sur les définitions, Deuxième cours international de criminologie consacré à la đangerosité. Impr. Melun, 1953. 
c'était une notion sans valeur scientifique 4 . On nous l'a reproché, et il importerait de voir d'une manière plus précise ce que cela pourrait vouloir dire. Dans le prolongement de cette discussion, nous chercherons à préciser de quelle manière plus directe (ou plus neutre), nous pourrions rejoindre cette préoccupation que suscite "la probabilité d'infraction". Cette manière plus directe et plus neutre nous parait d'ailleurs indispensable lorsque nous avons affaire à un comportement aussi complexe que ne l'est le comportement humain.

\section{LA DANGEROSITÉ, CONSTRUIT CRIMINOLOGIQUE}

\section{FAIT À PARTIR DE L'ÉTABLISSEMENT}

D'UN SCHÉMA CAUSAL

\section{DANS LE CADRE D'UN OBJECTIF POURSUIVI}

L'intitulé de ce premier point pourrait paraître banal, car, en effet, le fait qu'il y ait, dans une démarche scientifique, mise en lumière d'un lien causal et ce, en vue de réaliser un objectif déterminé, pourrait fort bien ne poser aucun problème. Nous pourrions d'ailleurs commencer notre analyse en banalisant au maximum les particularités de la notion de dangerosité par rapport à d'autres notions qui pourrait donner lieu à élaboration scientifique.

a) La criminologie a constitué, à sa naissance, un effort en vue d'introduire une perspective scientifique dans la manière d'aborder le problème de la délinquance. Lorsque nous parlons d'une perspective scientifique, nous voulons dire par là que les premiers criminologues ont cherché à comprendre le comportement délinquant à partir d'un schéma causaliste. C'est-à-dire que le comportement délinquant étant la variable dépendante, il est important de voir, à travers une analyse des relations ou des corrélations, quels étaient les facteurs susceptibles de le produire (ou d'en être la cause). L'utilisation par les auteurs de l'époque de la notion de dangerosité est directement liée à cette perspective et traduit en quelque sorte cette mise de la délinquance en «objet scientifique» à travers l'étude des conditions nécessaires au passage à l'acte et l'évaluation de la probabilité d'un comportement délinquant futur. L'objectif poursuivi, clairement exprimé à travers les notions de défense sociale et de dangerosité, est la maîtrise des activités criminelles et la gestion des populations susceptibles de poser problème à l'ordre social.

b) Il faut admettre qu'en faisant de la notion de dangerosité une notion charnière à travers laquelle le phénomène criminel allait être

4. La notion de dangerosité et sa mise en cause. VIIJ journées internationales de criminologie, Gênes, avril 1981. Voir à ce propos, l'article de G. Houchon publié ci-après. 
abordé, la criminologie, en qualité de nouvelle "science", n'a fait qu'entériner un objectif qui, déjà depuis des siècles, s'inscrivait ou était inscrit dans diverses politiques en vigueur. La notion de dangerosité a, en effet, sa préhistoire. Comme l'ont souligné de nombreux auteurs ${ }^{5}$, certains groupes d'individus (principalement mendiants, vagabonds) ont été considérés comme catégories dangereuses du fait que le pouvoir en place ne pouvait que difficilement les contrôler et les maîtriser. Pour cette raison, ils firent l'objet de mesures lorsque la tension sociale devenait trop forte ou encore lorsque l'État avait besoin de main-d'œuvre.

La notion de dangerosité s'inscrit donc dans cette perspective. Elle n'est pas une notion qui aurait été construite à partir d'une préoccupation intellectuelle soucieuse d'expliquer un phénomène. Elle trouve son origine dans une volonté politique qui s'est clairement affirmée : celle de gérer une population d'individus posant problème, en vue de la discipliner et d'exercer sur elle un contrôle tantôt pour s'en protéger, tantôt pour la faire entrer dans l'économie du pays, tantôt pour l'utiliser comme moyen de réorienter l'agressivité du groupe social. Dans ce sens, on peut effectivement dire comme l'affirment Foucault ${ }^{6}$ et dans la suite Houchon ${ }^{7}$, que cette notion participe plus aux pratiques disciplinaires et aux questions de gestion politique qu'à un effort d'élaboration scientifique.

Cela ne nous empêche cependant pas de méconnaître qu'est apparue à un certain moment, avec la naissance de la criminologie, une volonté de donner un support scientifique à ces réactions de politique criminelle face à une délinquance vécue comme de plus en plus difficilement maîtrisable. C'est bien souvent de cette manière que l'œuvre des positivistes italiens est considérée. On ne voit d'ailleurs pas pourquoi une attitude scientifique ne pourrait pas venir se greffer sur (ou s'insérer dans) la poursuite d'un objectif déjà donné. Mais il faut reconnaître que surgissent alors un certain nombre d'interrogations.

5. Nous pourrions citer depuis M. Ancel dans ses conférences faites au cours du $2^{t}$ cours international de criminologie (1953) jusqu 'aux études plus circonscrites de $F$. Tulkens et $F$. Digneffe, "La notion de dangerosité dans la politique criminelle en Europe occidentale"; P. Landreville et M. Petrunik, "Le "délinquant dangereux" dans les législations nord-américaines" et $\mathbf{J}$.' Rico, "Les législations hispano-américaines de dangerosité sociale», dans : Ch. Debuyst (édit.) (1981), Dangerosité et justice pénale, déjà cité.

6. M. Foucault (1981), "L'évolution dans la notion d'individu dangereux dans la psychiatrie légale», dans Débat : le dompteur face à la dangerosité, Déviance et société, vol. $5, \mathrm{n}^{\prime \prime} 4$, p. 403-421.

7. G. Houchon (1981), "La situation dangereuse. Aspects micro-sociologiques", dans Ch. Debuyst (édit.) (1981), Dangerosité et justice pénale. déjà cité. 
c) Lorsque l'on parle d'un projet scientifique en vue d'atteindre un objectif déterminé, il est évidemment illusoire que l'élaboration de l'objet d'étude ne constitue pas une construction élaborée en vue d'atteindre l'objectif que l'on cherche à poursuivre; cet objectif, dès lors, déterminera le point de vue que l'on sera amené à prendre. Autrement dit, poser le problème de la compréhension du comportement délinquant en termes de dangerosité peut sans doute apparaître comme une manjère utile et efficace de poser le problème; mais elle aboutira nécessairement à une sélection des données et au choix d'un cadre interprétatif dont l'intérêt n'est pas d'atteindre un comportement dans sa complexité et dans la diversité des significations qu'il présente, mais dans les indices qu'il révèle et qui, liés à d'autres indices, permet de croire à la dangerosité du sujet, ou à sa récidive.

L'impact de ce point de vue particulier sera considérable lorsque les instances qui le soutiennent ont à la fois le pouvoir de décision et dans une certaine mesure, le soutien de l'opinion publique, de telle sorte que ce point de vue constituera une "réalité contraignante".

En d'autres termes, nous voyons apparaître une césure entre les. données «utiles» ou politiquement utiles, et les données correspondant à une réalité effective et vécue.

On pourrait d'ailleurs aborder la question d'une autre manière : il est utopique de croire qu'une multiplication des connaissances et qu'une pluralité de points de vue (liée, par exemple à la pluridisciplinarité) s'imposent et auront pour conséquence une meilleure possibilité d'aborder ou de traiter un problème. En réalité, c'est le problème lui-même et la manière dont on l'aborde qui détermineront quels sont les connaissances et les points de vue intégrables dans la logique qui sous-tend cette manière de l'aborder. Ainsi, tout diagnostic de dangerosité nous impose déjà sa logique et nous enferme dans son point de vue. Il nous amène à ne prendre en considération que les informations utiles permettant de poser ce diagnostic. Multiplier les informations ou introduire des points de vue qui ne s'intègrent pas dans cette perspective constituent des opérations inutiles et même déroutantes (au sens où elles «déroutent» de l'objectif). C'est dans ce sens qu'effectivement, "les systèmes et les organisations n'ont besoin, pour fonctionner, que d'un savoir émietté» (M. Guillaume $)^{8}$ qui répond aux besoins précis définis par les préoccupations

8. J. Mandelbaum : entretien avec Marc Guillaume dans Économie, science inhumaine. Pour Marc Guillaume, les sciences sociales ont permis aux sociétés libérales de traiter l'homme comme un animal, journal le Monde, 22 et 23 avril 1984. 
de gestion. il en découle, comme le souligne le même auteur, que ce filtrage du savoir détermine des zones d'ignorance, ou plus exactement, traduit une volonté de maintenir des zones d'ignorance. Il s'agit là, dit-il, d'une fonction sociale importante à laquelle la science participe ${ }^{9}$. En d'autres termes, aborder l'étude des données criminologiques ou des comportements délinquants à travers la notion de dangerosité nous situe dans un système qui à la fois organise des connaissances partielles, utiles dans la mise en lumière de liens de causalité qu'elles semblent faire apparaître, et dans le même mouvement organise l'ignorance en mettant en dehors du champ tout ce qui pourrait rendre le savoir recueilli difficilement utilisable dans le cadre de l'objectif. On peut comprendre que le fait de maintenir des zones dignorance constitue une fonction sociale importante et que se pose à ce niveau un problème politique ${ }^{10}$.

d) Nous pourrions donner deux exemples qui constituent des illustrations (mais non des raisons) susceptibles de soutenir cette analyse.

Le premier nous réfère à un des premiers cours internationaux qui s'est déroulé à Bruxelles'". À cette occasion, avait eu lieu une discussion sur le thème : "importe-t-il d'élaborer un dossier de personnalité et importe-t-il de le transmettre avant ou après le jugement». Parmi les éminents criminologues présents, E. De Greeff fut le seul à soutenir que la prise en considération de ce dossier devait prendre place après le jugement. L'ensemble des autres lui objectaient en toute logique qu'un tel dossier n'avait de sens que dans la mesure où il donnait au juge les éléments lui permettant de prendre la décision la meilleure. La réponse du criminologue belge était que, dans le système de justice tel qu'il existait, les données que ce dossier fournirait au juge paralyserait au contraire toute possibilité de prendre une décision. En effet, s’il était sérieusement fait, ce dossier introduirait une complexité là où celle-ci n'était pas intégrable par le système auquel il était destiné. Pour le devenir, les données devraient être prédéterminées et réduites à un point tel qu'elles ne présenteraient plus aucun intérêt au niveau

9. J. Mandelbaum, op. cit. Cette idée apparaît d'ailleurs chez de nombreux auteurs pour ne citer que M. Sahlins (1980) dans sa Critique de la sociobiologie, Paris, NRF ou M. Serres (1983), Rome, le livre des fondations, Paris, Grasset, p. 81 («on n'a jamais vraiment évalué le prix de méconnaissance que suppose, que demande, que produit la connaissance").

10. Nous trouvons depuis Kepler des critiques faites à I'université d'Être, paradoxalement, la gardienne de l'ignorance (voir Mandelbaum et Guillaume, op. cit.).

11. Cycle européen de Bruxelles, 1953. 
d'une connaissance nuancée de ce qui s'est passé, ainsi que du point de vue des uns et des autres.

Ce jugement est sans doute sévère et il est probable que certaines informations préalables peuvent rendre les décisions en justice plus adéquates, tout comme une appréciation de la dangerosité (entendue au sens large) a sans doute pu constituer un progrès dans la prise en compte de certaines données. Ces progrès néanmoins sont limités dans la mesure où ils ne peuvent se faire qu'à l'intérieur d'une logique ainsi mise en mouvement, qui trouve son origine dans l'objectif disciplinaire ou de gestion des "groupes indisciplinés". Une information trop complexe perd donc son sens et n'est pas susceptible d'atteindre le niveau d'utilité dans une prise de décision.

Déjà à l'intérieur de la logique "dangerosité», une certaine complexité n'est pas intégrable dans l'utilisation de la notion. Le deuxième exemple que nous voudrions donner, en effet, est celui des réponses fournies par les criminologues engagés dans le travail professionnel à la question posée par l'équipe de recherche de l'Université de Montréal ${ }^{12}$ visant à savoir de quelle manière ils procèdent lorsqu'ils doivent se prononcer sur le potentiel de dangerosité de leur client. Ils en reviennent aux indices comportementaux, c'est-à-dire la nature du délit, les antécédents, les liens avec le milieu, l'intégration dans le monde du travail, etc., soit un exemple d'indices qui étaient ceux auxquels se référaient déjà les tables de prédiction de Burgess (1929) et qui font état des «habitudes prises». Cela paraît sommaire, mais relativement fiable, parce que cette appréciation se réfère à la notion psychologique d'habitude et des lieux où les habitudes se prennent (ou ne se prennent pas) et s'entretiennent. Il est possible que lorsque la question leur est posée de cette manière, les criminologues-praticiens n'aient pas grand-chose d'autre à faire; ils soulignent d'ailleurs la très grande difficulté de poser un diagnostic de dangerosité plus nuancé. La question est cependant celle de savoir - et nous revenons à ce que nous avons dit au début de cet article - pourquoi s'interroger sur la dangerosité et non pas sur l'habitude? En réalité, s'interroger sur la dangerosité suppose que l'on fasse simplement de l'habitude (et des éléments qui s'y réfèrent) un critère de dangerosité. Il faut reconnaittre qu'on ne s'interroge quasi pas sur l'habitude elle-même, sur ce qu'elle est et sur ce qu'elle veut dire.

12. J. Dozois, M. Lalonde et J. Poupart (1981), "La dangerosité : un dilemme sans issue? Réflexion à partir d'une recherche en cours», Déviance et société, vol. $5, \mathrm{n}^{\circ} 4$, p. $383-402$. 
C'est à ce niveau que nous pourrions dire que la notion de dangerosité détermine la manière dont les données doivent être connues (ou traitées), ainsi que les manières dont elles doivent être ignorées ou mises entre parenthèses. Nous pourrions le voir plus en détail à propos d'un point précis : l'apport multidisciplinaire en criminologie.

\section{LE CARACTÈRE MULTIDISCIPLINAIRE DE LA CRIMINOLOGIE COMME MYTHE OU COMME RÉALITÉ}

Les restrictions qu'apporte dans la connaissance d'un phénomène une perspective établie à priori détermine l'optique que l'on a en fonction de l'objectif que l'on poursuit et, pour cette raison, ne laisse qu'une place fort ambiguë à cette opération de mise en cause des connaissances (ou de mise à l'épreuve) que Popper considère comme essentielle dans les progrès de la connaissance. Le recours à la multidisciplinarité, par les interrogations qui en résultent, pourrait opérer de telles mises en cause. Ce que nous montrerons, dans le paragraphe qui suit, est la difficulté qu'a une volonté multidisciplinaire de pouvoir s'affirmer dans une criminologie dominée par la notion de dangerosité, sans se dénaturer et sans réduire sa portée. Ce fait ne constitue sans doute pas un problème spécifique et rejoint l'idée de Guillaume ${ }^{13}$ selon laquelle une étude multidisciplinaire suscite des interrogations telles que le retour au cloisonnement apparait souvent comme la seule solution.

a) Comment pouvoir le montrer dans le cadre d'une discipline, la criminologie, qui, par définition, parait être multidisciplinaire? Nous le ferons en partant d un exemple qui remonte à la criminologie du XIX ${ }^{e}$ siècle, auquel nous nous sommes déjà référés ${ }^{14}$, mais d'une manière qui n'était sans doute pas adéquate parce que cet exemple a donné lieu à de mauvaises interprétations qui ont fait parler de «sociologisme» ou d" "idéologie politique" à propos de ce qui n'était considéré que comme un arrière-fond à la criminologie clinique.

Notre point de départ se retrouve dans l'ouvre du Belge Ducpétiaux, qui, au moment de l'industrialisation naissante, a particulièrement étudié les rapports de la pauvreté et de la délinquance ainsi que les répercussions de l'urbanisme sur la délinquance, principa-

13. Dans Mandelbaum, op. cit.

14. Dans «La notion de dangerosité et sa mise en cause», VIII" journées internationales de criminologie clinique, op. cit. 
lement du phénomène des taudis, lié à l'afflux des travailleurs dans les grandes villes et à la manière dont les promoteurs de l'époque avaient résolu ce problème ${ }^{15}$. Pour expliquer la délinquance et les dangers d'une évolution vers la délinquance, Ducpétiaux, comme d'ailleurs d'autres auteurs, avait établi sa fameuse chaîne causale visant à mettre l'accent sur l'importance des taudis : taudis = apparition du dégoût de rester à la maison après le travail $=$ fréquentation des cafés et alcoolisme $=$ absentéisme au travail $=$ délinquance.

Nous n'avons pas à nous interroger sur le point de savoir si cette chaîne causale présente un support statistique. Ce serait effectivement important et une telle analyse révélerait toute l'ambiguïté des maillons qui la constituent. Elle présente en tout cas une certaine cohérence logique facilement compréhensible. Ce qu il nous importe de voir est la manière (ou les manières) dont le problème de la dangerosité est posé à travers cette "chaîne».

b) Un premier point est que la dangerosité naît de la conscience que l'on en a, et que cette conscience est déterminée par la position que l'on occupe face au phénomène donné. Cette prise de conscience comme celle de la chaîne causale explicative - pourra se faire par deux canaux différents : le canal individuel, c'est-à-dire le fait de rencontrer, dans la pratique des interventions judiciaires, des sujets délinquants dont le comportement va de pair avec l'alcoolisme, la méconduite, un désengagement professionnel se déroulant dans le cadre de la situation ouvrière, etc. Lorsque nous disons : "le fait de rencontrer", cela veut dire que ceux qui rencontrent, ou qui se heurtent à ce problème, font partie d'une catégorie sociale (juges, médecins, administrateurs pénitentiaires, etc.) qui ne se trouvent pas engagés dans cette aventure ouvrière, mais voient les divers éléments constitutifs de la situation à distance et à partir de préoccupations liées à l'ordre sociétaire. Nous avons ensuite le deuxième canal : le canal socio-politique; pour un gestionnaire politique comme l'était par exemple Ducpétiaux, la venue de certains événements sociaux sont suceptibles de mettre l'accent sur une réalité sociale jugée «explosive»; ainsi les taudis, qui seront le point de départ (comme à Bruxelles) d'épidémies qui risquent de se propager en dehors des délimitations de cette zone, le point de départ également de révoltes, le lieu où se localisent l'alcoolisme et un absentéisme de plus en plus marqué mettant en danger la viabilité des

15. Voir M. Smets (1977), l'Avènement de la cité jardin en Belgique, Histoire de l'habitat social en Belgique de 1830 à 1930, Bruxelles, Édit. Mardaga. 
entreprises, etc. Dans ce cas, la prise de conscience et le point d'entrée dans la chaîne causale est autre : il est localisé «dans» un phénomène social (ou dans une situation jugée dangereuse) dont les répercussions se traduiront à la fois au niveau social et au niveau individuel.

Voyons maintenant chacun de ces canaux et la manière dont les exigences multidisciplinaires se posent.

c) Le canal individuel par lequel s'établit une chaîne causale repose essentiellement sur la liaison entre la délinquance et certains indices comportementaux : milieux fréquentés (cafés) et conséquences qui en résultent (alcoolisme), non fréquentation professionnelle, etc. C'est-à-dire que nous retrouvons les indices auxquels se réfêrent les criminologues praticiens québécois, mais cette fois reliés à une réalité sociale spécifique et dans une perspective qui est celle de l'époque. De la même manière, le fait de lier la déinsertion professionnelle et familiale à la délinquance est finalement du même ordre que les explications de la délinquance données par Hirschi ${ }^{16}$ et selon lesquelles de tels sujets n'ont pas été en mesure de maintenir les liens dans lesquels s exprimeraient leurs engagements dans le monde du travail, le monde familial, etc.; ils devenaient "libres" pour tomber dans l'alcoolisme, dans la délinquance, etc., selon une logique qui parait effectivement constituer un support pour une compréhension de ces comportements.

Nous pourrions faire deux remarques qui prolongent ce que nous venons de dire. Dans cette perspective, il est facile d'introduire une forme de raisonnement multidisciplinaire qui s harmonisera avec la perspective prise et l'objectif poursuivi. Nous pourrions en effet nous référer à des facteurs sociaux comme les taudis, la misère, etc., ou encore à des explications psychiatriques qui, à l'époque, seront axées sur les conséquences de l'alcoolisme, c'est-à-dire la dégénérescence (Morel). On pourrait même complexifier les analyses psychologiques susceptibles de servir d'explication. D'autre part, c'est là notre deuxième remarque —, l'ensemble de ces explications vient tout naturellement s'intégrer dans une analyse de la "personnalité actuelle " ${ }^{17}$ mais une personnalité réduite à n'être qu'un objet d'étude permettant de comprendre la faille comportementale dont

16. T. Hirschi (1974), Causes of Delinquency. University of California Press.

17. Nous retrouvons ici les prises de position de J. Pinatel et de sa notion de personnalité criminelle, ainsi que celle de M. Fréchette. Nous aurions à foumir ici une bibliographie abondante. 
elle est le siège. Cette réduction objectiviste est essentielle dans cette perspective, et elle existe malgré l'utilisation de questionnaires à travers lesquels le "vécu" actuel du sujet pourrait être atteint ${ }^{18}$. Il s'agit en effet d'un vécu reconstruit par rapport à cette problématique de base : comprendre objectivement la faille comportementale, c'est-à-dire, la dangerosité du sujet. Derrière cette "personnalité actuelle", on a l'impression de voir se conjuguer la neurophysiologie et la psychiatrie, la psychologie et la sociologie, et d'avoir affaire à une perspective multidisciplinaire.

En quoi cette plurisdisciplinarité nous paraît-elle fictive? Elle l'est, nous semble-t-il, parce que, dans cette situation précise, elle ramène les préoccupations des diverses disciplines à un présupposé de départ qui est la «dangerosité» et son appréciation. Elle ne permet pas aux logiques propres de ces diverses disciplines de se développer et de mettre en cause ce présupposé de départ (qui d'ailleurs est un présupposé politique, ou un présupposé gestionnaire). Il y a à la fois une réalité sociale et une réalité psychologique qui n'est pas prise en compte, qui est ignorée ou occultée par la manière même dont le problème est posé.

Réalité sociale : le sujet n'est pas pris comme être social, c'est-àdire, comme être faisant partie d'une certaine classe sociale, située dans un rapport conflictuel, qui, à l'époque, subissait un véritable écrasement dont la réalité des taudis est en quelque sorte la manifestation. En d'autres termes, ce délinquant-ivrogne-travailleur irrégulier - et même dégénéré n’est pas seulement un sujet-objet qui présente ces caractéristiques du fait de l'intervention de facteurs sociaux (comme la misère et les taudis) ou de facteurs psychologiques. Le réduire à cela en fait effectivement, pour la criminologie conçue selon le modèle de la dangerositê, un objet-scientifique. Mais en réalité, celle-ci le reconstruit comme objet-maniable dans le cadre de l'objectif poursuivi. Ce pourrait évidemment être utile pour un gestionnaire dans une société donnée. Mais cela suppose l'élimination de tout un discours que le sujet pourrait tenir sur la situation qu'il connait, sur les injustices qu'il dit avoir vécues, sur l'impossibilité ressentie de faire valoir ses difficultés et ses griefs face aux

18. Nous abordons ici la question de la valeur des questionnaires. Voir par exemple Wegner et Vallacher (1977), Implicit Psychology. An Introduction to Social Cognition, New York, Oxford University Press, 326 p., ou encore J.C. Deschamps (1977), l'Attribution et la catégorisation sociale, Berne, Francfort-sur-Main, Ubl. Univ, europ., ou finalement, J.P. Leynes (1983), Sommes-nous tous des psychologues? Approche psychosociale des théories implicites de la personnalité, Bruxelles, Édit. Mardage, «Psychologie et Sciences humaines, " 
diverses autorités auxquelles il se trouvait confronté dans une relation de dépendance, etc. Dans une perspective que nous pourrions appeler sociologique, un tel discours serait interprété comme émanant d'un individu représentatif d'une position sociale, à l'intérieur d'une sociêté où les rapports de reconnaissance sont d'un certain type dont le discours de l'individu est porteur. C'est-à-dire que le sens de son discours (et par le fait même, dans une certaine mesure, ses caractéristiques de personnalité et les indices comportementaux) nous réfère à un contexte interprétable en termes tels qu'ils feraient "éclater" le schéma logique dans lequel les problèmes criminologiques ont été posés à partir de la notion de dangerosité. C'est en réalité ce qu'impliquerait une véritable interdisciplinarité ${ }^{19}$. Le problème pourrait être envisagé dans les mêmes termes pour l'être psychologique, car ce nous semblerait être une erreur que de n'envisager la psychologie dite criminelle qu'à l'intérieur de ce schéma logique de la dangerosité.

d) Nous pourrions prendre l'autre versant à partir duquel la chaîne causale présentée par Ducpétiaux pourrait être atteinte : le canal socio-politique. Ici encore, pour qu'il y ait dangerosité, il faut qu'it y ait prise de conscience, et prise de conscience à partir d'une position qui se situe en dehors de ce qui «cause» le danger. Certains événements mettent l'accent sur la signification dangereuse que présente une situation pour le groupe social et plus particulièrement pour le groupe social dominant, c'est-à-dire celui qui profite des structures sociales en place. Un conglomérat d'habitations (des taudis) peut devenir une situation de danger s'ils se produisent à partir de ces lieux des épidémies, des révoltes, etc. Une telle situation a des répercussions individuelles (qui sont décrites dans la chaîne) causale que cherche à dégager Ducpétiaux), mais pour les administrateurs ou les politiciens éclairés (comme l'était Ducpétiaux, secrétaire général au ministère de la Justice), la réaction souhaitée et considérée comme efficace se situe directement au niveau du phénomène jugé "cause» de la situation de danger, c'est-à-dire le taudis comme source de désintégration individuelle et de désintégration sociale. La politique proposée deviendra une politique de l'habitat qui ne vise pas seulement à donner un logement décent à ceux qui n'en avait pas; elle vise à inverser la relation causale, et c'est ici que nous retrouvons le problème disciplinaire. Tout comme le taudis est responsable du non-travail, de l'alcoolisme et de la désintrégation de

19. Voir C. Debuyst (1982), "L'interdisciplinarité considérée comme rupture d'un schéma d'interprétation", Revue interdisciplinaire d'études juridiques, n" 8, p. 123-130. L'ensemble du numéro est consacré à l'interdisciplinarité. 
toute responsabilité sociale, une politique de l'habitat ouvrier, habilement menée, devra viser à orienter ou à réorienter celui-ci vers une intégration dans le milieu du travail perçue comme nécessaire, vers une vie ordonnée et une stabilité sociale ${ }^{20}$. Cette préoccupation est constante dans toutes les discussions qui se sont déroulées sur ce thème au cours de la deuxième moitié du XIX $\mathrm{X}^{\mathrm{E}}$ siècle et la première du $\mathrm{XX}^{\mathrm{c}}$. Nous nous contenterons de citer une phase de Ducpétiaux prononcée au cours d'un débat sur la propriété des maisons ouvrières (1852) : "rendre propriétaire le travailleur ou le prolétaire, comme on l'appelle, c'est en faire un homme d'ordre, un père de famille rangé et laborieux...". En d'autres termes, la politique proposée en réaction au danger que présentait la situation sociale liée au caractère défectueux de l'habitat, repose sur l'établissement d'une relation causale inverse favorisant l'instauration d'une discipline dans le cadre de l'ordre existant.

Ici encore, nous pourrions nous demander à quel type d'interdisciplinarité nous avons affaire. Elle existe, car il est tenu compte des données individuelles et des facteurs socio-politiques en interaction les uns avec les autres. Mais pouvons-nous dire que le schéma "dangerosité" ne bloque pas tout ce qui ne s'intègre pas dans sa logique propre? Ici encore, nous pouvons croire qu'une véritable interdisciplinarité viendrait faire "éclater" ce cadre.

Un premier lieu d'éclatement se situerait au niveau individuel et psychologique, mais à un niveau qui ne serait pas schématisé dans la relation causale “alcoolisme - retrait du travail - comportement délinquant - éventuellement dégénérescence - éventuellement aussi, caractéristiques psychologiques de désengagement. Que pourrions-nous apprendre au niveau individuel, dans la mesure où nous n'enfermons pas les données que nous voulons recueillir dans la logique causale que nous venons de décrire? Il se peut très bien qu'à l'intérieur de ces taudis, certaines familles aient pu retrouver, ou aient pu constituer un équilibre relationnel et une possibilité d'expression culturelle (dans laquelle prennent place la délinquance et la révolte) liée à ce type de vie particulier, aux solidarités qui y sont possibles, avec les manifestations spécifiques qui y ont cours et qui donnent à ce groupe une réalité sociale qui ne sera décrite en termes négatifs que du point de vue de ceux qui ne sont préoccupés que par la bonne gestion d'un ordre social établi. Ou encore, il se peut aussi que certaines familles, face à l'angoisse de vie, aient trouvé dans ces taudis une sorte de refuge qui constituerait dans ce cas un équilibre jugé

20. Voir M. Smets, déjà cité (15). 
régressif mais autour duquel se cristallisent toutes les réactions de défense, de sorte qu'une attention particulière devrait pouvoir y être portée. Une politique qui consisterait à raser ces taudis pour les remplacer par des H.L.M. ou qui viserait à disséminer les habitants dans l'ensemble de la ville aurait sans doute pour elle, apparemment, le fait de résoudre le problème social que cet ilôt insalubre constituait, mais ne l'aurait fait qu'en ne tenant compte que d'une optique limitée à un seul niveau d'interprétation et axée sur une préoccupation déformante, c'est-à-dire exclusive de tout ce qui n'entre pas ou met en cause sa logique propre.

Un deuxième lieu d'éclatement se situerait évidemment au niveau d'une analyse socio-politique plus large : mettre l'accent sur cette analyse causale considérée comme suffisamment explicative élimine les facteurs politiques qui se situent au niveau de la structure sociétaire, elle-même susceptible de faire comprendre pourquoi le phénomène de taudisation est apparu et s'est développé, dans quel type de logique sociale, et également pourquoi la solution de l'habitat ouvrier, qui tend à être proposé comme solution, se situe dans une logique complémentaire. En d'autres termes, l'accent mis sur cette relation causale et la manière d'en tenir compte "partialise" le phénomène envisagé ou encore le transforme en un problème de gestion se situant dans un ordre sociétaire donné. Cette démarche nous paraît insatisfaisante, pour le moins au niveau d'une explication. Nous l'avons déjà souligné en parlant du "canal individuel". Mais se pose alors la question de savoir : dans le cadre d'une perspective individuelle comme dans le cadre d'une intervention sociale restreinte comment tenir compte de cette situation sociale plus globale, ou de ce que certains ont appelé la "violence de la société» 21 ?

\section{L'ÉCLATEMENT DE LA «DANGEROSITÉ» COMME NOTION ET COMME OUTIL. ÉBAUCHE D'UNE PERSPECTIVE}

Cette dernière question est la plus complexe. Elle consiste à dire : que faire encore avec cette notion de dangerosité dans une préoccupation qui serait celle d'atteindre à une connaissance plus exacte de la réalité criminelle, et qui en même temps ne négligerait pas les situations concrètes auxquelles le criminologue praticien se trouve confronté? On pourrait faire les propositions suivantes.

21. Voir Casadamont (1981), "Criminologie de la violence ou violence de la criminologie", Actions et recherches sociales, n" 1-2, p. 81 . 
a) La notion de dangerosité devrait être écartée comme cadre d'ensemble dans lequel prend place la réflexion criminologique. Elle introduit un à priori de départ et n'interprète les données qu'en fonction d'un diagnostic particulier qu'il importe de poser et qui repose sur des préoccupations politiques. (Celles-ci peuvent avoir un sens, mais elles doivent être définies comme telles.)

C'est la raison pour laquelle il nous paraît inadéquat d'étendre la définition de la dangerosité (probabilité de commettre une infraction) à une autre définition qui engloberait la démarche de connaissance qui serait celle du clinicien, par exemple. Ce fut le fait des criminologues cliniciens des années 50-60 qui avaient adopté la signification très vaste que Laignel-Lavastine avait donné à ce terme : «l'étude complète et intégrale de l'homme avec la préoccupation constante de mieux connaître les causes et les remèdes à une activité anti-sociale " ${ }^{22}$. Ce serait rendre ambiguë cette démarche de connaissance en la situant dans un cadre prédéterminé qui écarterait d'emblée les données qui seraient susceptibles de mettre ce cadre de référence en cause.

b) Lorsque l'on parle de "violence de la société" comme donnée situationnelle plus large dans laquelle les divers comportements prennent place, est-ce introduire une confusion de niveau, ou une prise de position idéologique? Canepa fait remarquer que ce qui intéresse la criminologie est la violence de l'individu et non la violence de la société, et par le fait même la dangerosité de l'indivi$\mathrm{du}^{2.3}$. Nous répondrons à cela que la violence de la société peut être un des éléments constitutifs de la violence de l'individu, et que dans le cadre même d'une relation ou d'une intervention clinique (ou d'une démarche de connaissance), cette éventualité ne peut d'aucune manière être exclue. Faire ainsi éclater la notion de dangerosité ne résulte nullement d'une politisation de la démarche du psychologue ou d'une naïveté qui consisterait, pour le clinicien, à vouloir prendre la violence sociétaire comme «objet». Elle résulte d'une perspective réellement interdisciplinaire, qui consiste à refuser de croire que cette violence institutionnelle (ou sociétaire) (et qu'il importerait d'ailleurs de décrire à tous les niveaux) n'intervient que d'une manière lointaine et illusoire qu'il importerait d'ignorer dans le cadre de l'examen (voir les fonctions sociales de l'ignorance).

22. Voir O. Kinberg (1959), les Problèmes fondamentaux en criminologie, Paris, Éd., Cujas, p. 138.

23. Cité par Casadamont, déjà cité. 
Cette dimension, au contraire, peut fort bien constituer un des ressorts à partir desquels le vécu prend sa signification, vécu lié non seulement aux infractions commises, mais aussi à la manière dont la réaction sociale à cette infraction s'exerce ou s'est exercée.

C'est à ce point de vue que la fameuse règle des niveaux d'interprétation, que $\mathrm{J}$. Pinatel a soulignée à maintes reprises ${ }^{24}$, tout en restant utile, révèle ses limites. Cette règle reste très utile en ce sens qu'elle évite d'opérer l'erreur importante qui consiste à tirer des conclusions irrecevables en opérant d'un niveau à l'autre. La limitation, cependant, consiste à réduire le type de lecture que l'on fait à un seul système logique dans lequel il paraît possible d'opérer (système, en l'occurrence dominé par la notion de dangerosité), et d'exclure dès l'abord tout ce qui est susceptible de faire éclater ce système, tout ce qui pourrait obliger de réordonner les données que l'on a rassemblées en fonction d'une autre logique qui rendrait ces données utilisables d'une manière non prévue.

Si problème politique il y a - (et nous rejoignons une nouvelle fois l'entretien déjà cité de Marc Guillaume) --, il consiste à refuser le jeu de cette fonction sociale qui consisterait à maintenir l'ignorance (à en devenir les gardiens) et à opérer dans ce but les cloisonnements qui permettraient d'éviter toute interrogation.

c) Un dernier point consisterait à parler des «outils». La notion de dangerosité est considérée comme un outil utile au moyen duquel il est possible d'approcher un problème donné. Il est manifestement, à ce point de vue, surévalué. Sans qu'on ne puisse s'étendre sur cette question (et qui vaudrait la peine d'un autre numéro), on peut dire que, ce qui nous importerait au niveau d'une pratique, c'est en venir à des notions «neutres» (dans le sens où l'entend Ball Rokeach ${ }^{25}$ ). La notion de dangerosité n'est pas pas une notion "neutre". Elle implique d'emblée un point de vue qui aboutit à ne voir un individu qu'à travers les critères à partir desquels sa dangerosité pourrait être établie. Adopter des critères neutres ne veut pas dire que l'on rejette toute idée selon laquelle une situation ou une interrelation pourrait devenir explosive et qu'il ne serait pas possible de le prévoir; ou encore qu'un individu ne présente pas une probabilité plus grande qu'un autre de commettre une infraction. Mais de tels faits doivent

24. J. Pinatel (1963), Traité de droit pénal et de criminologie (Bouzat et Pinatel), t. III, Criminologie, Paris, Éd. Dalloz, p. 52.

25. S.J. Ball-Rokeach (1980), "Normative and Deviant Violence from a Conflict Perspective", Social Problems, vol. 28, n" 1, p. 45-61. 
être resitués dans une perspective plus large; il importe d'intégrer dans leur compréhension la pluralité des acteurs en cause et la pluralité des sens possibles que ces faits sont susceptibles de prendre.

Pour pouvoir le faire, il nous paraît d'abord nécessaire de rejeter un critère d'analyse qui unifierait toutes les situations comme le fait la notion de dangerosité. Il importerait de les distinguer comme de distinguer les problèmes en adaptant la démarche suivie à chaque type de problème et à ses particularités. On pourrait dire : parcelliser ou distinguer les problèmes, mais ne pas morceler les connaissances qui s'y réfèrent, ne pas les "émietter" selon les exigences d'une gestion (ou les insuffisances d'une gestion) qui généralisera forcément. À partir de là, nous voyons émerger différents points.

Nous avons d'abord la notion d'habitude prise, la récidive pouvant se considérer comme une habitude prise. Pourquoi identifier la récidive à la dangerosité et pas à une habitude prise? Ce que nous pourrions considérer comme "rendre une notion neutre" ce serait d'abord de dire, ou de se dire, que toute habitude qui s'est prise amène le sujet à recommencer le comportement dont il a pris l'habitude. Il s'agit là d'une règle générale, qui n'a rien d'exceptionnel ou d'étonnant et qui vaut pour celui qui a commis un acte délinquant comme pour les autres actes. L'objection que l'on pourrait alors faire est celle de dire qu'il s'agit d'une «mauvaise habitude" et que la sanction, ou que le conditionnement serait justement ce qui permettrait de rectifier cette «mauvaise orientation". Mais ici encore, on échappe à une analyse plus rigoureuse de l'habitude en la classant comme mauvaise (c'est-à-dire en opérant une généralisation), et en imaginant en même temps l'homme comme être dont la seule caractéristique est la malléabilité, ce qui semble loin d'être vrai (Tarde le disait déjà lorsqu'il notait, dans l'introduction de son ouvrage sur l'imitation, que toute imitation appelle une contre-imitation). C'est-à-dire que l'on pose immédiatement le problème en termes de "réaction à" sans voir le sens qu'a le phénomène de départ et en cherchant éventuellement à le réduire à ses causes (ce qui néglige le fait que nous ayons affaire à un sujet, c'est-à-dire, à un être qui a des projeț, qui a des désirs et non seulement des besoins, etc.).

Lorsque nous ouvrons le dictionnaire au terme habitude, nous voyons déjà apparaître, dans le vocabulaire courant, des différences liées au sens qu'a cette «manière de se comporter, fréquemment répétée» : habitude $=1$ ) usage d'une collectivité, d'un lieu (ce sont les habitudes de l'endroit, du pays), 2) usage répété, action répétée qui 
apporte l'habileté ou la connaissance (l'habitude du métier), etc. C'està-dire que I'on pourrait commencer par voir ce que les mots veulent dire dans leur utilisation courante, et ne poser le problème de l'interdit que dans un deuxième temps.

Ceci n'est qu'une perspective explicitée d'une manière très sommaire. Nous en aurions d'autres : les situations conflictuelles ou des interrelations conflictuelles et la manière dont des négociations peuvent se nouer. Nous pourrions ainsi continuer et il serait intéressant de le faire. La seule conclusion que nous voudrions tirer est celle qui préconise un éclatement de la notion de dangerosité, qui, à première vue, parait recouvrir une réalité évidente, mais qui, à seconde vue, est beaucoup plus de l'ordre d'une préoccupation qui unifie artificiellement une pluralité de difficultés se situant dans des problématiques différentes et qui pourraient donner lieu à une pluralité de démarches. 